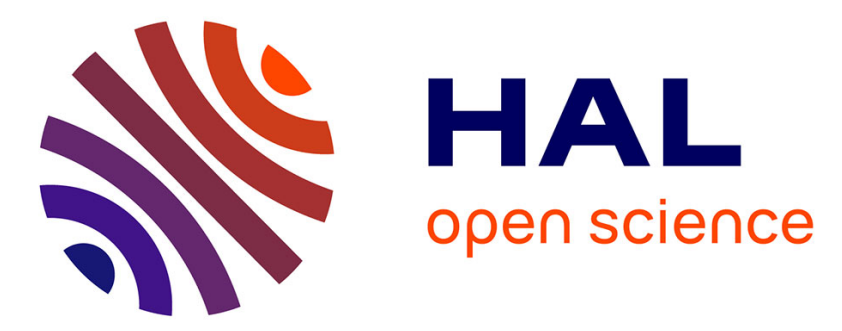

\title{
Biobased thermosensitive polyrotaxanes constructed by polymerization of cyclodextrin-triterpenoid inclusion complexes
}

\author{
Jiawei Li, Xia Yu, Yiran Zhao, Hao Zhang, Min-Hui Li, Jun Hu
}

\section{- To cite this version:}

Jiawei Li, Xia Yu, Yiran Zhao, Hao Zhang, Min-Hui Li, et al.. Biobased thermosensitive polyrotaxanes constructed by polymerization of cyclodextrin-triterpenoid inclusion complexes. Polymer Chemistry, 2020, 11 (40), pp.6492-6498. 10.1039/d0py00966k . hal-03052662

HAL Id: hal-03052662

https://hal.science/hal-03052662

Submitted on 10 Dec 2020

HAL is a multi-disciplinary open access archive for the deposit and dissemination of scientific research documents, whether they are published or not. The documents may come from teaching and research institutions in France or abroad, or from public or private research centers.
L'archive ouverte pluridisciplinaire HAL, est destinée au dépôt et à la diffusion de documents scientifiques de niveau recherche, publiés ou non, émanant des établissements d'enseignement et de recherche français ou étrangers, des laboratoires publics ou privés. 
Check for updates

Cite this: Polym. Chem., 2020, 11, 6492

Received 5th July 2020,

Accepted 8th September 2020

DOI: $10.1039 / \mathrm{d} 0$ py00966k

rsc.li/polymers

\section{Biobased thermosensitive polyrotaxanes constructed by polymerization of cyclodextrin- triterpenoid inclusion complexes $\uparrow$}

\begin{abstract}
Jiawei Li, ${ }^{a}$ Xia Yu, ${ }^{a}$ Yiran Zhao, ${ }^{a}$ Hao Zhang, ${ }^{a}$ Min-Hui Li (D) a,b and Jun Hu (D) *a
In this work, three cyclodextrin-based polyrotaxanes (CD-based PRs) with alternating multiblock structures were constructed by polymerization of inclusion complexes in a convenient tandem way, where an inclusion complex of a natural glycyrrhetinic acid (GA) derivative and $\gamma$-CD polymerized with three PEGdicarbonates, respectively, followed by capping with amino- $\beta-C D$. During the formation of $C D-$ based PRs, only a small amount of $\gamma$-CD slipped off and around $90 \%$ of GA units on the guest polymer were complexed with $\gamma$-CD. Note that although the three CD-based PRs had similar molecular weights of 50 $000 \mathrm{Da}$, the decrease of the PEG length (from 4000 to $600 \mathrm{Da}$ ) led to an enhancement of the structural rigidity and the variation in the hydrophilic-lipophilic balance (HLB). In this case, the sizes of the assembled spherical micelles and the reversible phase transition behaviors were effectively regulated by the molecular rigidity and HLB. Our biocompatible CD-based PRs with precise controllable performance may lead to further exploration of thermosensitive materials.
\end{abstract}

\section{Introduction}

Taking advantages of unique topological features and stimuliresponsive functions, mechanical interlocking molecules (MIMs) consisting of supramolecular structures have broad application prospects in the fields of drug/gene delivery, tissue engineering, and molecular devices. ${ }^{1-3}$ As one of the typical MIMs, polyrotaxanes (PRs) are constructed by threading cyclic host molecules on a linear guest polymer axle with bulky end caps, and their components can generate relative motions to achieve the desired functions driven by dynamic and reversible non-covalent interactions. ${ }^{4}$ Among them, cyclodextrin (CD)-based PRs have received increasing research attention because they are important molecular models in understanding macromolecular recognition in biological systems such as

\footnotetext{
${ }^{a}$ Beijing Advanced Innovation Center for Soft Matter Science and Engineering, Beijing University of Chemical Technology, North Third Ring Road 15, Chaoyang District, Beijing 100029, China.E-mail: jhu@mail.buct.edu.cn

${ }^{b}$ Chimie ParisTech, PSL University Paris, CNRS, Institut de Recherche de Chimie Paris, 11 rue Pierre et Marie Curie, Paris 75005, France

$\dagger$ Electronic supplementary information (ESI) available: Materials and methods; preparation and characterization of amine- $\beta$-CD, GA-derivatives, PEG-dicarbonates, CD-based PRs; calculation of the structural parameters of CD-based PRs; MS and XRD spectra of the GA-diamine $/ \gamma$-CD inclusion complex; ${ }^{1} \mathrm{H}$ NMR, $2 \mathrm{D}$ NOESY of PR-2000 and PR-600; XRD, SEC, statistical particle sizes, SLS, and CMC results of PR-4000, PR-2000, and PR-600; particle size distribution of PR-600 aqueous solutions; transmittance of PR-2000 aqueous solutions with cooling-heating cycles. See DOI: 10.1039/d0py00966k
}

antigen-antibody and enzyme-substrate pairs. ${ }^{5,6}$ Compared with other synthetic cyclic molecules like calixarenes, pillararenes and cucurbiturils, cyclodextrins are produced by enzymatic hydrolysis of starch with good biocompatibility and biodegradability, and are a class of macrocyclic oligosaccharides with truncated cone structures. ${ }^{7,8}$ The abundant hydroxyl groups on the outer surface are easy to be modified for imparting functionality, while the hydrophobic inner cavity allows CDs to accommodate multifarious guest molecules to form stable inclusion complexes and assemble into supramolecular architectures. $^{9-11}$ To date, hydrophilic polyethers like polyethylene glycol (PEG) and polypropylene glycol (PPG) have been widely used as guest polymers in the fabrication of biocompatible CD-based PRs. ${ }^{12}$ However, the aggregation between these threaded cyclodextrins often reduces the uniformity of polyrotaxanes, thus causing the lack of precise control of their supramolecular structures. Moreover, the simple repetitive chemical structure and monotonous functionalization of these guest polymers limit the development of CD-based PRs in structures and functions. ${ }^{13-15}$ Therefore, searching for a suitable guest main-chain polymer of CD-based PRs with diverse structures and controllable conformations remains an open question.

Many well-known guest molecules like azobenzene, ferrocene and adamantane are often utilized in the construction of advanced CD-based PRs which exhibit excellent potential in self-healability and stimuli-responsiveness. ${ }^{16-18}$ Nevertheless, these guest molecules are either toxic or lack reactive sites, and are not suitable building blocks to fabricate biocompatible main- 
chain CD-based PRs. Glycyrrhetinic acid (GA), a classic natural triterpenoid molecule, not only has good biocompatibility and biodegradability, but also possesses a hydrophobic rigid skeleton and reactive groups at the head and tail positions, which facilitate the introduction of GA into polymer main chains. ${ }^{19}$ More importantly, it can form a stable host-guest complex with $\mathrm{CD}^{20,21}$ Using these GA-CD host-guest pairs, self-healable supramolecular hydrogels have been successfully prepared in our previous works. $^{22}$ Obviously, GA would be an ideal guest molecule in the construction of biocompatible CD-based PRs.

Herein, in this work we developed three CD-based PRs (PR-4000, PR-2000, PR-600) via polymerization of inclusion complexes in a convenient tandem way (Scheme 1). Initially, the inclusion complex was prepared using $\gamma$-CD and a GA derivative (GA-diamine), which subsequently condensed with PEG-dicarbonate to afford polypseudorotaxanes with alternating structures. Finally, by capping with amine- $\beta$-CD, CD-based PRs were obtained successfully (for synthetic details, see the ESI $\dagger$ ). In these three CDbased PRs, all components are biocompatible, and their molecular rigidity and hydrophilic-lipophilic balance (HLB) can be adjusted by changing the length of the PEG spacer for the regulation of the CD-based PR conformation. The results showed that: (1) alternating multiblock supramolecular architectures were constructed, where $\gamma$-CDs specifically recognized GA units in PR-4000, PR-2000, and PR-600 with a high complexation ratio of around 90\%; (2) PR-4000, PR-2000, and PR-600 had similar number-average molecular weights $\left(M_{\mathrm{n}}\right)$ while they possessed different fractions of hydrophobic and hydrophilic segments, which allowed their structural rigidity and assembly behavior to be easily regulated; and (3) PR-4000 and PR-2000 exhibited temperature-dependent phase behaviors in aqueous solution, and their phase transition temperatures can be tuned by the HLB of PRs. Our work provides a simple yet convenient strategy for constructing biobased thermosensitive main-chain type CD-based PRs with precise controllable performance, which may benefit their potential applications in thermosensitive materials.

\section{Results and discussion}

\section{Synthesis and characterization of an inclusion complex between GA-diamine and $\boldsymbol{\gamma}$-CD}

The hydroxyl and carboxyl groups at the head and tail positions of GA allow it to easily access the backbone of mainchain type guest polymers. Initially, GA reacted with 2-bromoethanol to afford GA-diol, which was confirmed by the

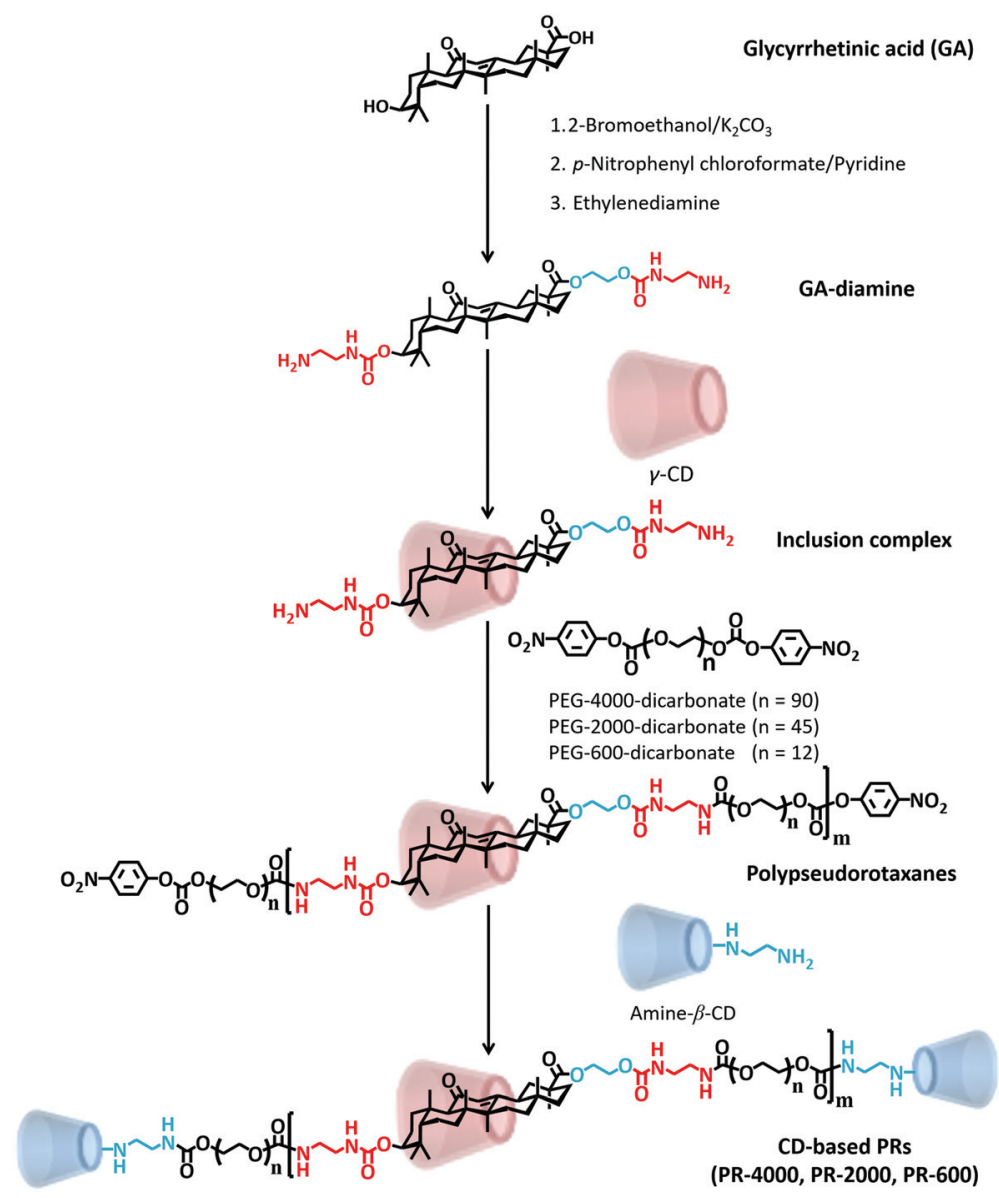

Scheme 1 Synthesis of main-chain type CD-based polyrotaxanes constructed by CD-GA pairs via polymerization of inclusion complexes in a convenient tandem way. 
appearance of proton peaks at 4.17-4.33 and 3.83-3.85 ppm assigned to methylene groups in the ${ }^{1} \mathrm{H}$ NMR spectrum $\left(\mathrm{H}_{31}\right.$ and $\mathrm{H}_{32}$, Fig. S1A $\dagger$ ). Subsequently, $p$-nitrophenyl chloroformate was introduced to activate the hydroxyl groups of GA-diol to give GA-dicarbonate. Due to the generation of carbamate bonds, the proton peaks of $\mathrm{H}_{31}, \mathrm{H}_{32}$, and $\mathrm{H}_{3}$ shifted downfield from 4.21, 3.84 , and $3.23 \mathrm{ppm}$ to $4.51,4.51$, and $4.40 \mathrm{ppm}$, respectively, accompanied by the appearance of peaks at 7.41 and $8.27 \mathrm{ppm}$ of $p$-nitrophenol $\left(\mathrm{H}_{35}\right.$ and $\mathrm{H}_{36}$, Fig. $\left.\mathrm{S} 1 \mathrm{~B} \dagger\right)$. Finally, GA-dicarbonate was ammonolysed to GA-diamine with proton signals of the amine ester bond $\left(\mathrm{H}_{\mathrm{a}}\right.$ and $\mathrm{H}_{\mathrm{b}}$ at 5.89 and $\left.5.02 \mathrm{ppm}\right)$ and methylene groups $\left(\mathrm{H}_{\mathrm{c}}\right.$ and $\mathrm{H}_{\mathrm{d}}$ at 3.25 and $\left.2.83 \mathrm{ppm}\right)$ as shown in Fig. S1C. $\uparrow$ That is to say, two amino groups were successfully introduced into the head $\left(\mathrm{C}_{3}\right.$-position $)$ and tail $\left(\mathrm{C}_{30}\right.$-position $)$ of GA for the production of GA-diamine.

After that, GA-diamine was allowed to form the inclusion complex with $\gamma$-CD. Compared with the sharp proton signals of $\gamma$-CD in $\mathrm{D}_{2} \mathrm{O}$ (Fig. 1A), only weak broad peaks of $\mathrm{H}_{9}, \mathrm{H}_{12}$, and methyl groups $\left(\mathrm{H}_{23-29}\right)$ from GA-diamine were observed in $\mathrm{D}_{2} \mathrm{O}$ in the ${ }^{1} \mathrm{H}$ NMR spectrum because of its hydrophobic nature (Fig. 1B). Upon complexing with $\gamma$-CD, all proton peaks from GA-amine sharpened and increased. The peak signals of $\mathrm{H}_{9}$ and $\mathrm{H}_{12}$ shifted downfield from 1.87 and $5.42 \mathrm{ppm}$ to 2.29 and $5.61 \mathrm{ppm}$ promoted by hostguest interactions between GA and $\gamma$-CD (Fig. 1C). By calculating the proton integration ratio of $\mathrm{H}_{12}$ from GA and $\mathrm{H}_{1}$ from $\gamma$-CD, the complexation ratio of GA-diamine with $\gamma$-CD was approximately $1: 1$. 2D nuclear Overhauser enhancement spectroscopy (NOESY) gave more direct results as shown in Fig. 1C, where the appearance of NOE correlations between $\mathrm{H}_{3} / \mathrm{H}_{5}$ of $\gamma$-CD and methyl groups of GA-diamine identified their spatial contacts, strongly demonstrating that the GA moiety penetrated into the cavity of $\gamma-\mathrm{CD}^{23,24}$ Moreover, $\mathrm{H}_{9}, \mathrm{H}_{12}$ and methyl peaks of the GA-diamine $/ \gamma$-CD inclusion complex were observed at chemical shifts different from that of the free GA-diamine, which clearly indicated that GA-diamine formed a stable complex with $\gamma$-CD without free GA-diamine (Fig. S2 $†$ ). This was consistent with the results of mass spectrometry (Fig. S3†), where the molecular ion peak of the inclusion complex of GAdiamine $/ \gamma-\mathrm{CD}\left(M_{\mathrm{w}} 1983\right)$ was observed with the disappearance of the molecular ion peaks of GA-diamine $\left(M_{\mathrm{w}} 686\right)$ and $\gamma$-CD $\left(M_{\mathrm{w}} 1297\right)$. More evidence for the formation of the GA-diamine $/ \gamma$-CD inclusion complex came from the X-ray diffraction (XRD) pattern. GA-diamine showed an amorphous structure (Fig. S4A $\dagger$ ), and $\gamma$-CD revealed a series of characteristic peaks at $12.3^{\circ}, 15.3^{\circ}, 16.5^{\circ}, 18.7^{\circ}$ and $23.4^{\circ}$ (Fig. S4B $\dagger$ ), confirming the "cage-type" crystalline structure. ${ }^{25}$ When equimolar amounts of GA-diamine and $\gamma$-CD were mechanically mixed, the diffraction pattern was just the superimposition of the two components (Fig. S4C $\dagger$ ). Conversely, the inclusion complex was classified as a tetragonal channel structure with the characteristic diffraction peaks at $2 \theta=11.6^{\circ}$ and $16.1^{\circ}$ (Fig. S4D †). ${ }^{26}$ All the above results strongly illustrated the formation of the stable inclusion complex of GA-diamine $/ \gamma$-CD.

\section{Preparation and characterization of CD-based PRs via polymerization of inclusion complexes}

CD-based PRs with amphiphilic alternating structures were obtained by polycondensation of the inclusion complex with

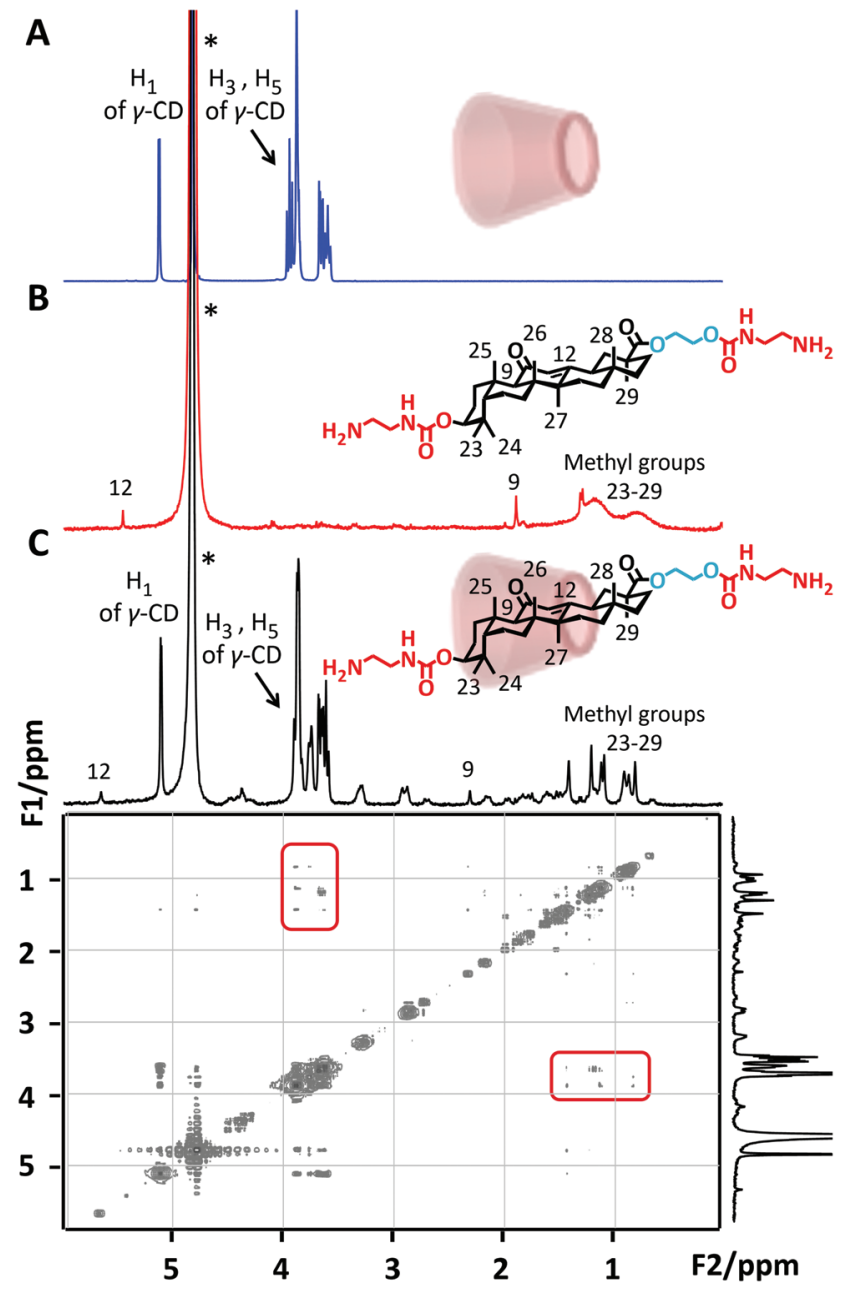

Fig. $1{ }^{1} \mathrm{H}$ NMR spectra of (A) $\gamma-C D$ and (B) GA-diamine in $\mathrm{D}_{2} \mathrm{O}$ at $25^{\circ} \mathrm{C}$ (400 MHz). (C) ${ }^{1} \mathrm{H}$ NMR and $2 \mathrm{D}$ NOESY spectra of the inclusion complex of GA-diamine $/ \gamma-\mathrm{CD}([\mathrm{GA}] /[\gamma-\mathrm{CD}]=1)$ in $\mathrm{D}_{2} \mathrm{O}$ at $25{ }^{\circ} \mathrm{C}(600 \mathrm{MHz})$. * Represents the solvent peak (correlation signals are marked by red rectangles).

PEG-dicarbonate (PEG-4000, PEG-2000, PEG-600), followed by the reaction with the terminating group of amino- $\beta$-CD. Note that a slight excess of PEG-dicarbonate was required for facilitating the capping of amino- $\beta$-CD with terminal carbonate groups. Taking PR-4000 as an example, upon polycondensation, the proton peak of $\mathrm{H}_{34}$ on GA-diamine at $2.88 \mathrm{ppm}$ in Fig. 2C shifted to 3.26 ppm in Fig. 2A, while the peak of $\mathrm{H}_{\mathrm{c}}$ on PEG-4000-dicarbonate in Fig. 2B upshifted to $4.23 \mathrm{ppm}$ in Fig. 2A, all of which were attributed to the formation of amine ester bonds in PR-4000. The complete disappearance of the peaks of $\mathrm{H}_{\mathrm{a}}$ and $\mathrm{H}_{\mathrm{b}}$ of $p$-nitrophenyl groups further revealed the successful capping of polyrotaxanes (Fig. 2A). The NOE correlation of protons from methyl groups on GA with the interior $\mathrm{H}_{3} / \mathrm{H}_{5}$ of $\gamma$-CD in PR-4000 is shown in Fig. 2D, similar to the results of the inclusion complex as shown in Fig. 1C, strongly confirming that $\gamma$-CD recognized GA units in PR-4000. XRD measurements were also employed to provide more structure information of CD-PRs in the solid state. As shown in 


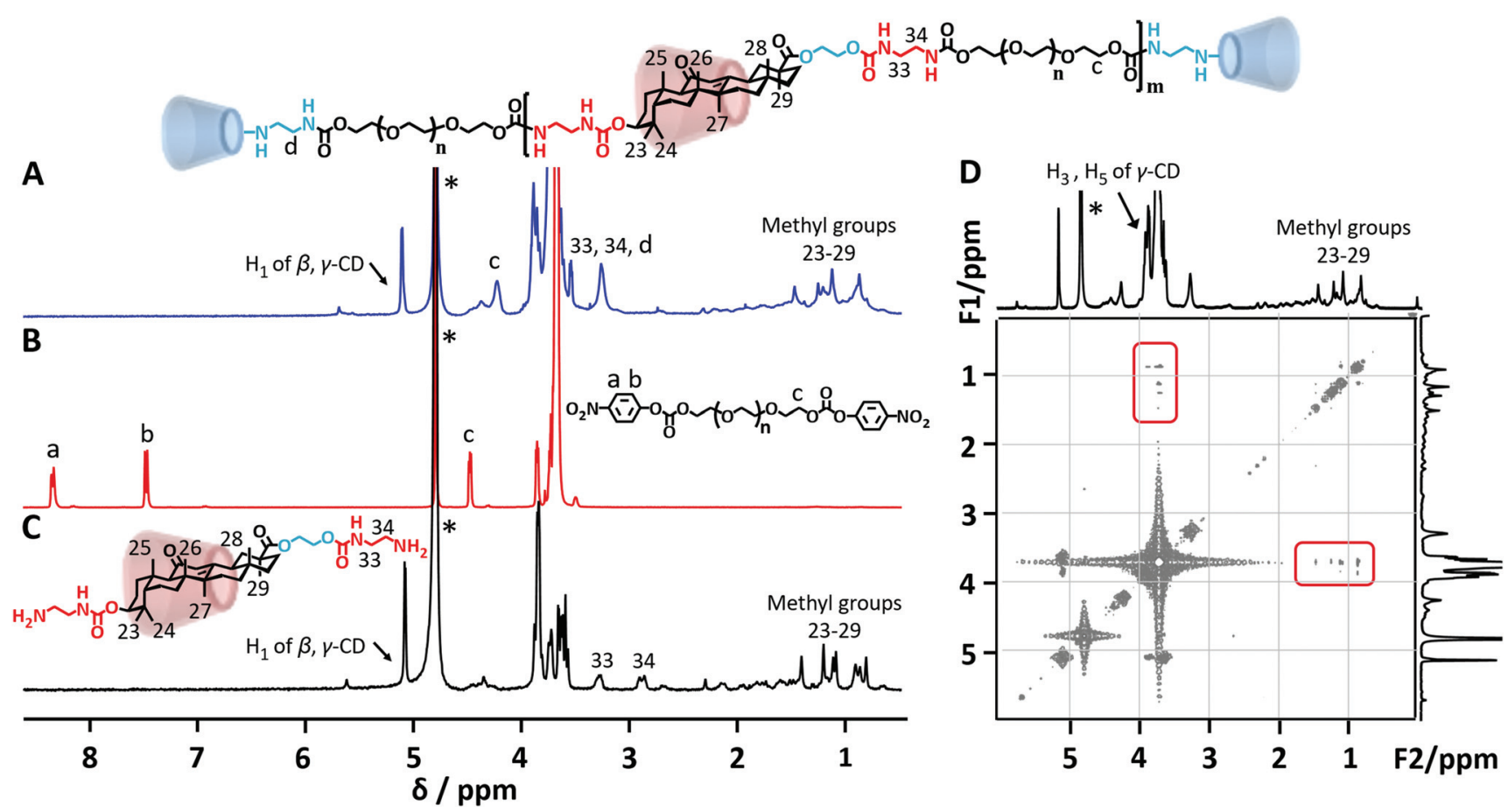

Fig. $2{ }^{1} \mathrm{H}$ NMR spectra of (A) PR-4000, (B) PEG-4000-dicarbonate, and (C) the inclusion complex of GA-diamine with $\gamma$-CD (400 MHz, $\mathrm{D}_{2} \mathrm{O}$ ). (D) 2D NOESY spectrum of PR-4000 in $\mathrm{D}_{2} \mathrm{O}$ at $25^{\circ} \mathrm{C}(600 \mathrm{MHz})$. * Represents the solvent peak (correlation signals are marked by red rectangles).

Fig. S5A, $\uparrow$ diffraction peaks at $19.0^{\circ}$ and $23.2^{\circ}$ belonging to the PEG-4000 chain were observed, whereas the diffraction peaks of the inclusion complex GA-diamine $/ \gamma$-CD disappeared completely, indicating that $\gamma$-CD selectively recognized GA units without the formation of a "channel-type" structure with PEG segments. ${ }^{27,28}$ Similar to PR-4000, the structures of PR-2000 and PR-600 with different PEG spacers have also been characterized by XRD (Fig. S5B and $\mathrm{C} \dagger$ ), ${ }^{1} \mathrm{H}$ NMR (Fig. S6 and 7†), and 2D NOESY (Fig. S8 and 9†).

Combining the integration ratio of $\mathrm{H}_{33}, \mathrm{H}_{34}$, and $\mathrm{H}_{\mathrm{d}}$ versus $\mathrm{H}_{1}$ of $\mathrm{CD}$ in the ${ }^{1} \mathrm{H}$ NMR spectra (Fig. 2A) and the results of size exclusion chromatography (SEC) in Fig. S10, $\dagger$ the complexation ratio of $\gamma$-CD with the GA unit in CD-based PRs was calculated according to eqn (S1-3), $\dagger$ and the results are presented in Table 1 . The complexation ratio of $\gamma$-CD with the GA unit was estimated to be $90 \%, 87 \%$, and $92 \%$ in PR-4000, PR-2000, and PR-600, respectively, revealing the shedding of only a small amount of $\gamma$-CD during the formation of PRs. Moreover, although the $M_{\mathrm{n}}$ of the three PRs remained around $50000 \mathrm{Da}$, the number of GA on each PR chain gradually increased as the length of the PEG fragment decreased, consequently leading to an enhancement of the structural rigidity and the variation in the hydrophilic-lipophilic balance (HLB) of CD-based PRs.

\section{Assembly and thermosensitive behavior of CD-based PRs}

The difference in the structural rigidity and hydrophilicity of CD-based PRs affected the conformation and assembly morphology. Transmission electron microscopy (TEM), dynamic
Table 1 Structural parameters of CD-based PRs

\begin{tabular}{llll}
\hline Data & PR-4000 & PR-2000 & PR-600 \\
\hline$M_{\mathrm{n}}{ }^{a}$ & 56300 & 50200 & 49800 \\
$\Xi^{a}$ & 1.15 & 1.33 & 1.47 \\
Number of GA on each PR chain $^{b}$ & 8.5 & 11.9 & 18.7 \\
Complexation ratio $^{c}$ & $90 \%$ & $87 \%$ & $92 \%$
\end{tabular}

${ }^{a}$ Measured by SEC with DMF as an eluent against a polystyrene standard. ${ }^{b}$ Calculated from the integration ratio of $\mathrm{H}_{33}, \mathrm{H}_{34}$, and $\mathrm{H}_{\mathrm{d}}$ versus $\mathrm{H}_{1}$ of $\mathrm{CD}$ in the ${ }^{1} \mathrm{H}$ NMR spectra, and the molecular weight of CD-based PRs in SEC. ${ }^{c}$ Defined as the ratio of GA units complexed by $\gamma$-CD to the total GA units in PRs.

light scattering (DLS) and static light scattering (SLS) measurements were performed to explore the self-assembly of CD-PRs in water. As shown in Fig. 3A-C, all three CD-based PRs assembled into spherical micelles, and their diameters followed an order of PR-2000 (12 nm) < PR-4000 (29 nm) < PR-600 (55 nm) according to the statistical results (Fig. S11†). This order was consistent with the DLS results as shown in Fig. 3D, where the average hydrodynamic diameter was 15, 50, and $91 \mathrm{~nm}$ for PR-2000, PR-4000, and PR-600, respectively. Note that the particle sizes from the TEM images were smaller than those from the DLS results, which was mainly because of the dehydration of micelles during the TEM sample preparation. ${ }^{29}$ These results can be further confirmed by SLS measurements. Taking PR-4000 as an example, the gyration radius $\left(R_{\mathrm{G}}\right)$ was $35 \mathrm{~nm}$ as shown in Fig. S12. $\dagger$ Accordingly, the structure-sensitive parameter $\left(\rho=R_{\mathrm{G}} / R_{\mathrm{H}}\right)$ was equal to 1.4 being compatible 
A

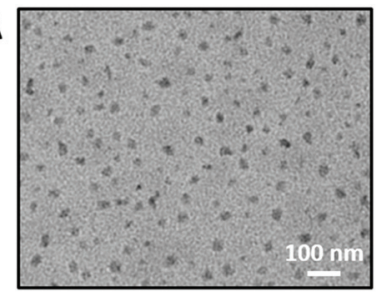

C

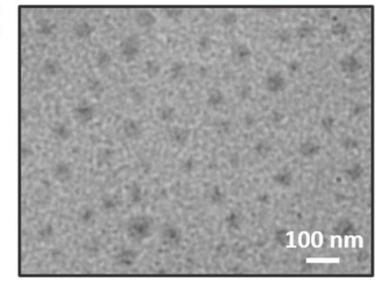

B
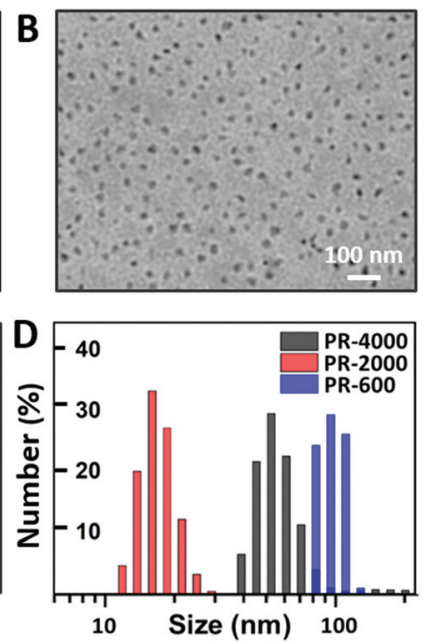

Fig. 3 TEM images of the aqueous solution of (A) PR-4000, (B) PR-2000, and (C) PR-600, and (D) their particle size distributions from the $\mathrm{DLS}$ results. Concentration: $2.5 \mathrm{mg} \mathrm{mL}^{-1}$.

with the spherical block copolymer micelle. ${ }^{30}$ As the molecular length of GA $(1.27 \mathrm{~nm})$ was larger than the height of $\gamma$-CD $(0.79 \mathrm{~nm})$, only a part of the GA unit could enter the cavity of $\gamma$-CD, which made the inclusion complex of CD-GA relatively hydrophobic in comparison with PEG fragments. Thus, the CD-based PRs were amphiphilic structures formed by alternating hydrophobic inclusion complexes and hydrophilic PEG fragments. ${ }^{22}$ In aqueous solution, the $\mathrm{CD}-\mathrm{GA}$ inclusion complexes cannot be folded because of their high rigidity, while they tended to aggregate with each other in a "bridge" conformation to form a hydrophobic core under hydrophobic forces. Meanwhile, the hydrophilic PEG fragments like to adopt a "loop" conformation by folding to form a hydrophilic layer for stabilizing the formed hydrophobic core, thus resulting in the formation of spherical micelle structures. ${ }^{31,32}$ In this case, the longer hydrophilic segment allowed PR-4000 to have a larger diameter than that of PR-2000. Unlike them, the significant decrease in the PEG length of PR-600 would cause a lack of hydrophilicity in the micellar shell, consequently fusing with each other to form larger micelles.

In addition, the critical micelle concentration (CMC) values for the three CD-based PRs were evaluated from the fluorescence emission spectrum with pyrene as the probe. ${ }^{33}$ As the ratio of the fluorescence intensity of pyrene at 373 and $384 \mathrm{~nm}$ was sensitive to the surrounding solvent polarity, it was plotted as a function of the concentrations of CD-based PRs. As shown in Fig. S13, $\dagger$ clear inflection points were observed, which revealed the formation of micelles with the respective CMC values of $4 \times 10^{-2}, 3 \times 10^{-2}$, and $3 \times 10^{-5} \mathrm{mg} \mathrm{mL}^{-1}$ for PR-4000, PR-2000, and PR-600. Clearly, the value of the CMC decreased as the proportion of hydrophobic components increased. $^{34}$

Furthermore, the alternating structures composed of PEG fragments and $\mathrm{CD}-\mathrm{GA}$ pairs endowed the aqueous solution of CD-based PRs with thermo-responsiveness, displaying a lower critical solution temperature (LCST) determined by temperature-variable UV-Vis spectroscopy. ${ }^{35}$ As shown in Fig. 4A, the clear solution changed to a turbid dispersion during the heating process and the typical LCST behavior was observed both for PR-4000 and PR-2000 with the phase transition temperature at 73 and $47{ }^{\circ} \mathrm{C}$, respectively. During the heating process, the hydrogen bonds between CD-based PRs and water molecules were weakened, while the ones between CD-based PRs formed more easily. In this case, the aggregation between hydrophobic cores of $\mathrm{CD}-\mathrm{GA}$ inclusion complexes was enhanced and the outer PEG shell tended to shrink or even collapse, consequently forming larger micelle aggregates. ${ }^{36,37}$ Conversely, no phase transition occurred for PR-600 that tended to aggregate in the test temperature range. This tendency in the LCST behavior was attributed to the decrease of PEG segments from PR-4000 to PR-2000, and PR-600. Moreover, the transmittance changes of PR-4000 and PR-2000 during two heating and cooling cycles were similar (Fig. 4B and S14 $\dagger$ ), revealing the reversibility of phase transitions and their thermostability. Note that a certain hysteresis in the phase transition temperature occurred during the cooling process, which was because the formation of aggregates at high temperature was accompanied by enhanced interactions between polymer chains, which delayed the disassembly of aggregates. ${ }^{38}$

In addition, the temperature-dependent DLS results further verified the thermal response behavior of CD-based PRs. As shown in Fig. 4C, the particle size of PR-4000 was around $70 \mathrm{~nm}$ in the temperature range of $25-70{ }^{\circ} \mathrm{C}$. When the temperature rose to $75{ }^{\circ} \mathrm{C}$, the particle size dramatically increased to around $400 \mathrm{~nm}$, which corresponded to the phase transition behavior of PR-4000 aqueous solution. Compared with PR-4000, the particle size of PR-2000 showed more significant dependence on the temperature (Fig. 4D). The initial particle size of PR-2000 was only $10 \mathrm{~nm}$ in the temperature range of
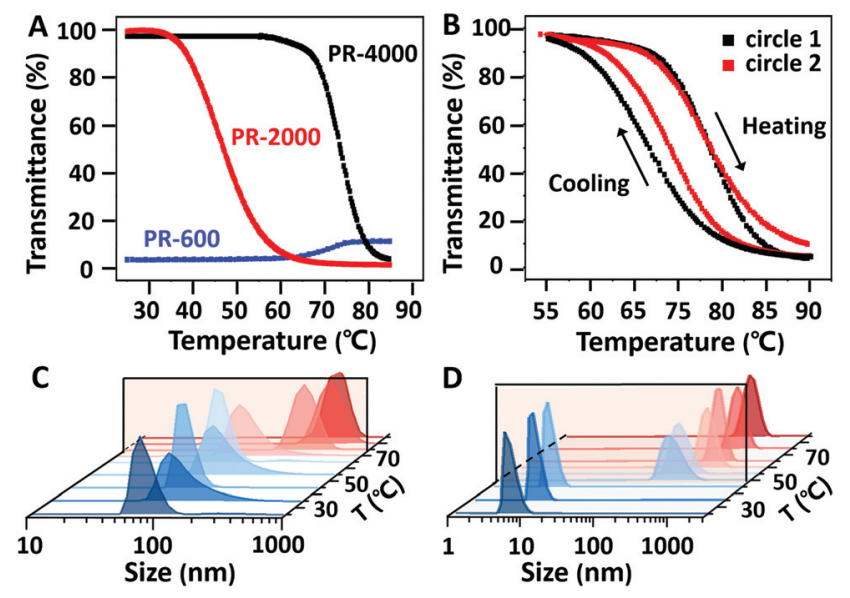

Fig. 4 Transmittance of aqueous solutions of CD-based PRs as a function of temperature at a wavelength of $600 \mathrm{~nm}$ : (A) PR-4000, PR-2000, and PR-600 at a concentration of $2.5 \mathrm{mg} \mathrm{mL}^{-1}$ from 25 to $85^{\circ} \mathrm{C}$ and (B) the aqueous solution of PR-4000 with two cooling-heating cycles. Particle size distribution of aqueous solutions of (C) PR-4000 and (D) PR-2000 from the DLS results in the temperature range from 25 to $85^{\circ} \mathrm{C}$. Concentration: $2.5 \mathrm{mg} \mathrm{mL}^{-1}$. 
$25-45{ }^{\circ} \mathrm{C}$. When the temperature reached $50{ }^{\circ} \mathrm{C}$, the particle diameter increased to $220 \mathrm{~nm}$, and then $500 \mathrm{~nm}$ with further temperature increase to $60{ }^{\circ} \mathrm{C}$. The temperature interval where the particle size changed agreed well with the results of the phase transition temperatures measured by UV-Vis spectrometry. It needs to be noted that the diameter of PR-600 remained at $\sim 130 \mathrm{~nm}$ without any LCST property in the same temperature range (Fig. S15 $\dagger$ ). The $D$ values of these particles at different temperatures are presented in Table S1. $\dagger$ Apparently, the assembly and thermosensitive phase behaviors of our CD-based PRs in water can be regulated effectively by adjusting the length of the PEG fragment.

\section{Cytotoxicity and protein adsorption of CD-based PRs}

MTT cytotoxicity assays were performed to evaluate the cytotoxicity of CD-based PRs. ${ }^{39}$ As shown in Fig. 5A, HepG2 cells retained about $90 \%$ viability after incubation for $24 \mathrm{~h}$ in the presence of CD-based PRs below a concentration of $100 \mu \mathrm{g}$ $\mathrm{mL}^{-1}$, indicating that the three CD-based PRs had no significant cytotoxicity. In addition, protein adsorption was another important factor for biocompatibility evaluation. ${ }^{40}$ Herein, bovine serum albumin (BSA) was used as a model protein to study the protein adsorption of CD-based PRs by measuring their hydrodynamic diameters before and after incubation with BSA in phosphate buffered saline (PBS). ${ }^{30}$ As shown in Fig. 5B-D, except for the overlap between PR-2000 and BSA, the distributions of PR-4000 and PR-600 were bimodal in the presence of BSA (the population centered at around $8.4 \mathrm{~nm}$ was related to free BSA). After incubation with BSA for $24 \mathrm{~h}$, no change in hydrodynamic diameters of CD-based PRs was observed, which meant no adsorption happened. Obviously, both cytotoxicity and protein adsorption assays indicated that all three CD-based PRs had good biocompatibility.
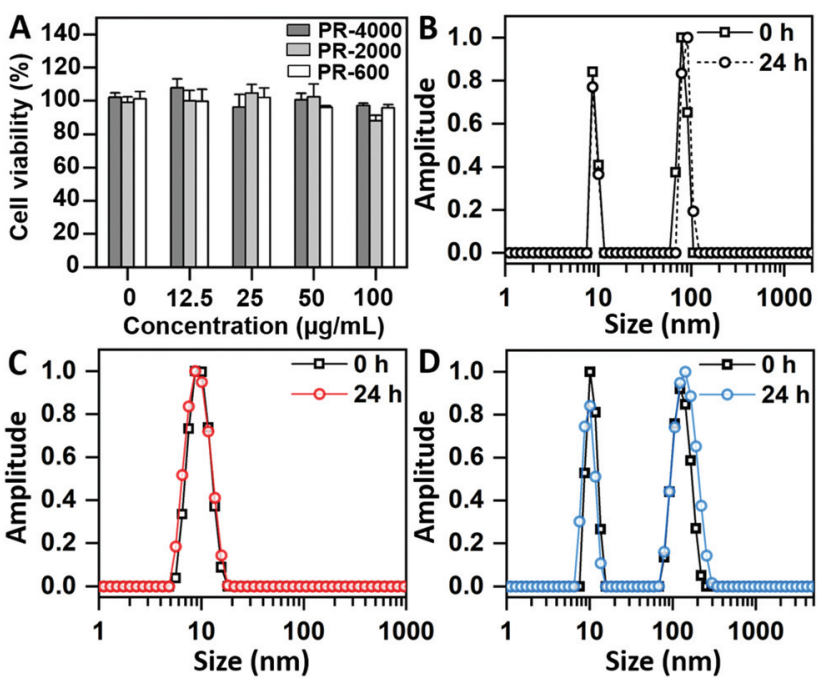

Fig. 5 (A) MTT cytotoxicity assay of CD-based PRs. Size distributions of PR-4000 (B), PR-2000 (C) and PR-600 (D) in the presence of BSA at $t=0 \mathrm{~h}(\square)$ and $t=24 \mathrm{~h}(\mathrm{O})$.

\section{Conclusions}

In summary, we prepared three biocompatible main-chain type CD-based PRs with different PEG spacers using cyclodextrin-triterpenoid pairs via polymerization of inclusion complexes in a convenient tandem way. Their amphiphilic alternating structures were characterized by $1 \mathrm{D} / 2 \mathrm{D}-\mathrm{NMR}, \mathrm{MS}, \mathrm{XRD}$, SEC, and TEM. The results showed that (1) $\gamma$-CD retained the specific recognition of GA units with a high complexation ratio of around $90 \%$ without any localization to PEG chains. Only $10 \%$ of $\gamma$-CD slipped off during the formation of CD-based PRs, reflecting the high efficiency of polymerization of inclusion complexes. Notably, $\gamma$-CDs were relatively evenly distributed on the main chain of the guest polymer without aggregation, and the structures of CD-based PRs were uniform and orderly, which avoided the influence of $\gamma$-CD aggregation on the performance of polyrotaxanes; (2) PR-4000, PR-2000, and PR-600 had the similar number-average molecular weights of $\sim 50000 \mathrm{Da}$, but their molecular rigidity increased as the proportion of hydrophobic GA fragments increased, which endowed them with different HLB values. The cyclodextrin coverage and HLB value can be controlled accurately by adjusting the length of PEG fragments, which thus facilitated the regulation of the assembly and thermal response performance of polyrotaxanes; and (3) all three CD-based PRs assembled into spherical micelles, and PR-4000 and PR-2000 exhibited reversible LCST behaviors in water. The dependence of the micelle size and phase transition temperature on the structural rigidity and HLB of CD-based PRs afforded a convenient way to tailor the assembly and thermosensitive phase behaviors. These CD-based PRs have potential applications in thermosensitive materials.

\section{Conflicts of interest}

There are no conflicts to declare.

\section{Acknowledgements}

This work is supported by the National Key R\&D Program of China (no. 2017YFD0200302) and NSFC (no. 21604085).

\section{References}

1 G. Yu, B. C. Yung, Z. Zhou, Z. Mao and X. Chen, ACS Nano, 2018, 12, 7-12.

2 C. J. Bruns and J. F. Stoddart, Acc. Chem. Res., 2014, 47, 2186-2199.

3 E. R. Kay and D. A. Leigh, Angew. Chem., Int. Ed., 2015, 54, 10080-10088.

4 W. Yang, Y. Li, H. Liu, L. Chi and Y. Li, Small, 2012, 8, 504516.

5 A. Harada, Y. Takashima and M. Nakahata, Acc. Chem. Res., 2014, 47, 2128-2140. 
6 E. Persch, O. Dumele and F. Diederich, Angew. Chem., Int. Ed., 2015, 54, 3290-3327.

7 R. Dong, Y. Zhou, X. Huang, X. Zhu, Y. Lu and J. Shen, Adv. Mater., 2015, 27, 498-526.

8 J. Szejtli, Chem. Rev., 1998, 98, 1743-1753.

9 V. R. de la Rosa and R. Hoogenboom, Chem. - Eur. J., 2015, 21, 1302-1311.

10 V. R. de la Rosa, P. Woisel and R. Hoogenboom, Mater. Today, 2016, 19, 44-55.

11 B. V. K. J. Schmidt and C. Barner-Kowollik, Angew. Chem., Int. Ed., 2017, 56, 8350-8369.

12 S. Chelli, M. Majdoub, S. Aeiyach and M. Jouini, J. Polym. Sci., Part A: Polym. Chem., 2009, 47, 4391-4399.

13 A. Tonegawa, A. Tamura and N. Yui, ACS Macro Lett., 2019, 8, 826-834.

14 S. Chelli, M. Majdoub, G. Trippé-Allard, S. Aieyach, K. I. Chane-Ching and M. Jouini, Polymer, 2007, 48, 36123615.

15 A. Hashidzume, H. Yamaguchi and A. Harada, Eur. J. Org. Chem., 2019, 3344-3357.

16 D. Taura, S. Li, A. Hashidzume and A. Harada, Macromolecules, 2010, 43, 1706-1713.

17 G. Rydzek, T. Garnier, P. Schaaf, J. Voegel, B. Senger, B. Frisch, Y. Haikel, C. Petit, G. Schlatter, L. Jierry and F. Boulmedais, Langmuir, 2013, 29, 10776-10784.

18 A. Bin Imran, K. Esaki, H. Gotoh, T. Seki, K. Ito, Y. Sakai and Y. Takeoka, Nat. Commun., 2014, 5, 5124-5431.

19 H. Du, X. Yang, X. Pang and G. Zhai, Carbohydr. Polym., 2014, 111, 753-761.

20 J. Hao, Y. Gao, Y. Li, Q. Yan, J. Hu and Y. Ju, Chem. - Asian J., 2017, 12, 2231-2236.

21 T. Ishida, I. Miki, T. Tanahashi, S. Yagi, Y. Kondo, J. Inoue, S. Kawauchi, S. Nishiumi, M. Yoshida, H. Maeda, C. Tode, A. Takeuchi, H. Nakayama, T. Azuma and S. Mizuno, Eur. J. Pharmacol., 2013, 714, 125-131.

22 Y. Li, J. Li, X. Zhao, Q. Yan, Y. Gao, J. Hao, J. Hu and Y. Ju, Chem. - Eur. J., 2016, 22, 18435-18441.

23 S. Saha, A. Roy, K. Roy and M. N. Roy, Sci. Rep., 2016, 6, 35764-35775.
24 V. T. Karathanos, I. Mourtzinos, K. Yannakopoulou and N. K. Andrikopoulos, Food Chem., 2007, 101, 652658.

25 T. Uyar, M. A. Hunt, H. S. Gracz and A. E. Tonelli, Cryst. Growth Des., 2006, 6, 1113-1119.

26 S. Zhang, M. Fan, Y. Liu, Y. Ma, G. Zhang and J. Yao, Langmuir, 2007, 23, 9443-9446.

27 X. Fu, L. Yang, J. Ma, G. Yang, Y. Yao and Q. Chen, Polymer, 2016, 105, 310-317.

28 I. N. Topchieva, A. E. Tonelli, I. G. Panova, E. V. Matuchina, F. A. Kalashnikov, V. I. Gerasimov, C. C. Rusa, M. Rusa and M. A. Hunt, Langmuir, 2004, 20, 9036-9043.

29 J. Logie, S. C. Owen, C. K. McLaughlin and M. S. Shoichet, Chem. Mater., 2014, 26, 2847-2855.

30 F. C. Giacomelli, P. Stepánek, V. Schmidt, E. Jäger, A. Jäger and C. Giacomelli, Nanoscale, 2012, 4, 45044514.

31 Q. Xu, S. Li, C. Yu and Y. Zhou, Chem. - Eur. J., 2019, 25, 4255-4264.

32 S. Li, K. Li, Q. Xu, Y. Wang, C. Yu and Y. Zhou, Phys. Chem. Chem. Phys., 2019, 21, 25148-25157.

33 J. Wang, Y. Song, P. Sun, Y. An, Z. Zhang and L. Shi, Langmuir, 2016, 32, 2737-2749.

34 X. Wu, X. Chen, P. Hu, M. Hou, Y. Dong and Y. Wei, Carbohydr. Polym., 2018, 191, 136-141.

35 B. Yeniad, K. Ryskulova, D. Fournier, J. Lyskawa, G. Cooke, P. Woisel and R. Hoogenboom, Polym. Chem., 2016, 7, 3681-3690.

36 J. Han, Y. Weng, J. Xu and B. Guo, Colloids Surf., A, 2019, 575, 84-93.

37 C. Zhao, Z. Ma and X. Zhu, Prog. Polym. Sci., 2019, 90, 269291.

38 H. Cheng, L. Shen and C. Wu, Macromolecules, 2006, 39, 2325-2329.

39 B. Saha, N. Choudhury, S. Seal, B. Ruidas and P. De, Biomacromolecules, 2019, 20, 546-557.

40 Z. Pan, D. Fang, N. Song, Y. Song, M. Ding, J. Li, F. Luo, H. Tan and Q. Fu, ACS Appl. Mater. Interfaces, 2017, 9, 2138-2149. 